\title{
Experimental Evidence for Limited in vivo Virulence of Mycobacterium africanum
}

\author{
Baltazar Cá1,2,3,4,5, Kaori L. Fonseca 1,2,3,4, Jeremy Sousa 1,2,4, Ana Raquel Maceiras 1,2, \\ Diana Machado6, Lilica Sanca5, Paulo Rabna ${ }^{5}$, Pedro N. S. Rodrigues ${ }^{1,2,4}$, \\ Miguel Viveiros ${ }^{6}$ and Margarida Saraiva ${ }^{1,2 *}$ \\ 1 i3S - Instituto de Investigação e Inovação em Saúde, Porto, Portugal, ${ }^{2}$ Instituto de Biologia Molecular e Celular, \\ Universidade do Porto, Porto, Portugal, ${ }^{3}$ Programa de Pós-Graduação Ciência para o Desenvolvimento, Instituto \\ Gulbenkian de Ciência, Oeiras, Portugal, ${ }^{4}$ Instituto de Ciências Biomédicas Abel Salazar, Universidade do Porto, Porto, \\ Portugal, ${ }^{5}$ Instituto Nacional de Saúde Pública/Projeto de Saúde de Bandim, Bissau, Guinea-Bissau, ${ }^{6}$ Global Health \\ and Tropical Medicine, Instituto de Higiene e Medicina Tropical, Universidade Nova de Lisboa, Lisbon, Portugal
}

\section{OPEN ACCESS}

Edited by: George Grant, University of Aberdeen, United Kingdom

Reviewed by: Leopold Tientcheu, Medical Research Council The Gambia Unit (MRC), Gambia

Isaac Darko Otchere,

Noguchi Memorial Institute for Medical Research (NMIMR),

Ghana

*Correspondence: Margarida Saraiva margarida.saraiva@ibmc.up.pt

Specialty section: This article was submitted to Infectious Diseases,

a section of the journa

Frontiers in Microbiology

Received: 09 July 2019

Accepted: 26 August 2019 Published: 10 September 2019

Citation:

Cá B, Fonseca KL, Sousa J, Maceiras AR, Machado D, Sanca $L$, Rabna P, Rodrigues PNS, Viveiros $M$ and Saraiva M (2019) Experimental Evidence for Limited in vivo Virulence

of Mycobacterium africanum.

Front. Microbiol. 10:2102.

doi: 10.3389/fmicb.2019.02102
Tuberculosis remains a public health problem and a main cause of death to humans. Both Mycobacterium tuberculosis and Mycobacterium africanum cause tuberculosis. In contrast to $M$. tuberculosis, which is geographically spread, $M$. africanum is restricted to West Africa. Differences have also been found in the growth rate and type of disease caused by $M$. africanum, globally suggesting an attenuation of this bacteria. In this study, we used the mouse model of infection to follow the dynamics of $M$. africanum infection in terms of bacterial burdens and tissue pathology, as well as the immune response triggered. Our findings support a lower virulence of $M$. africanum as compared to $M$. tuberculosis, including in mice lacking IFN- $\gamma$, a major protective cytokine in tuberculosis. Furthermore, the lung immune response triggered by $M$. africanum infection in wild-type animals was characterized by a discrete influx of leukocytes and a modest transcriptional upregulation of inflammatory mediators. Our findings contribute to elucidate the pathogenesis of $M$. africanum, supporting the hypothesis that this is an attenuated member of the tuberculosis-causing bacteria. Understanding the biology of $M$. africanum and how it interacts with the host to establish infection will have implications for our knowledge of TB and for the development of novel and better tools to control this devastating disease.

Keywords: tuberculosis, Mycobacterium africanum, immune response, cytokines, pathology

\section{INTRODUCTION}

Tuberculosis remains a global health problem, with approx. 10 million new cases and over 1.3 million deaths in 2017 (WHO, 2018). Both Mycobacterium tuberculosis and Mycobacterium africanum can cause human TB (Brites and Gagneux, 2015). These TB-causing bacteria are in turn divided into $7 \mathrm{~L}$ belonging to the MTBC, with L1, 2, 3, 4, and 7 encompassing M. tuberculosis sensu

Abbreviations: ${ }^{-/-}$, deficient; BMDM, bone marrow-derived macrophages; CFU, colony forming unit; DC, dendritic cell; ESAT6, early secreted antigenic target, $6 \mathrm{kDa}$; H\&E, hematoxylin and eosin; L, lineage; MOI, multiplicity of infection; MTBC, Mycobacterium tuberculosis complex; PBMCs, peripheral blood mononuclear cells; TB, tuberculosis. 
stricto, and L5 and 6 encompassing M. africanum (Gagneux, 2018). Relevant diversity at genomic, clinical and immunological level has been well documented for M. tuberculosis (Bastos et al., 2017). Recent studies show that diversity within M. africanum L5 and L6 also exists (Ates et al., 2018; Otchere et al., 2018), but its functional impact remains unknown. Interestingly, whereas infections with $M$. tuberculosis are globally widespread, those with $M$. africanum are geographically restricted to West Africa (Yeboah-Manu et al., 2017). Indeed, isolation of M. africanum from patients with $\mathrm{TB}$ in non-African countries has been mostly restricted to migrants from endemic areas in Africa (Isea-Pena et al., 2012).

The mechanisms underlying the restricted ecological niche of $M$. africanum remain elusive. This restriction may result from specific host-pathogen interactions, as supported by the association of $M$. africanum L5 with TB patients of the Ewe ethnicity in Ghana (Asante-Poku et al., 2015), and by the presence of specific mutations in the genes encoding the ESX secretion system in bacteria of L5 and L6, which could represent adaptations to the niche of the West African host (Winglee et al., 2016). Another possibility relies on $M$. africanum being zoonotic with an animal reservoir limited to West Africa. Infection of animals with M. africanum have been described (Thorel, 1980; Alfredsen and Saxegaard, 1992; Gudan et al., 2008), and a novel MTBC strain closely related to L6 has been isolated from a wild chimpanzee (Coscolla et al., 2013). However, evidence of person to person transmission of $M$. africanum has also been reported (Winglee et al., 2016), thus suggesting that an animal reservoir is not fully required. Finally, M. africanum may be an attenuated member of the MTBC, and consequently outcompeted by M. tuberculosis lineages, in most settings. This last hypothesis is supported by several lines of evidence. Epidemiologically, a decline on the incidence of $M$. africanum has been reported over time for some countries (Dosso et al., 1999; Kallenius et al., 1999; Koro Koro et al., 2013), although not for others (Gehre et al., 2013; Yeboah-Manu et al., 2017). At the clinical level, M. africanum has been associated with a slower progression to disease than M. tuberculosis (de Jong et al., 2008), and reported to be more commonly found in HIV, malnourished and older individuals (de Jong et al., 2010a). The preferential association of $M$. africanum with HIV remains, however debatable (Meyer et al., 2008). Furthermore, several M. tuberculosis key virulence mechanisms have been shown to be affected in $M$. africanum (Yeboah-Manu et al., 2017). Such is the case of the DosR regulon (Ofori-Anyinam et al., 2017), ESAT6 (de Jong et al., 2006), and PhoP/R (Gonzalo-Asensio et al., 2014). Although scarcely explored, data from experimental animal models also point to a slower progression of disease upon infection with $M$. africanum than that observed with M. tuberculosis (Bold et al., 2012; Wiens and Ernst, 2016b).

Understanding the course of infection of $M$. africanum, particularly the type of immune responses developed to this member of the MTBC as compared to M. tuberculosis sensu stricto, may shed light into novel strategies to tackle TB. In this study, we followed the progression of experimental (mouse) aerosol infection by a clinical isolate of $M$. africanum. As compared to $M$. tuberculosis infections, the clinical isolate of
M. africanum under study was associated with lower bacterial burdens, signs of disease and tissue pathology, even in hosts lacking IFN- $\gamma$, a critical protective molecule in TB. Moreover, the infection was accompanied by a modest cellular and molecular immune response in the lungs of infected mice. Our findings contribute to elucidate the pathogenic potential of $M$. africanum, supporting the hypothesis that $M$. africanum is a less virulent member of the TB-causing bacteria.

\section{MATERIALS AND METHODS}

\section{Ethics Statement}

Animal experiments were performed in strict accordance with the recommendations of the EU Directive 2010/63/EU and approved by the Portuguese National Authority for Animal Health (DGAV-Ref. 0421/000/000/2016). Mice were kept with food and water ad libitum and humanely euthanized by $\mathrm{CO}_{2}$ asphyxiation. Every effort was made to minimize suffering.

The human study that originated the blood samples for PBMC isolation was reviewed and approved by the Portuguese Comissão de Ética para a Saúde da ARS Norte (project T792). Written informed consent was obtained for collection of biological material and data from all study participants were anonymized.

\section{Animals}

Eight to twelve-week-old male and female C57BL/6 WT or IFN- $\gamma$ deficient $\left({ }^{-/}\right)$mice were bred and housed at i3S animal facility. For infections, animals were housed under contention conditions in the Animal Biosafety Level 3 facility at i3S.

\section{Bacteria Isolation and Characterization}

Mycobacterium africanum G67 (Maf_G67) was isolated in Guinea-Bissau at the Instituto Nacional de Saúde Pública (INASA) from a TB patient diagnosed and followed at the Raoul Follereau Hospital, Bissau, Guinea-Bissau, in 2012 (Rabna et al., 2015). It was grown on MGIT tubes for the BACTEC MGIT 960 (Becton-Dickinson, Sparks, MD, United States) according to the manufacturer's instructions. The Genotype MTBC assay (Hain Lifescience, Nehren, Germany) was used for the differentiation within the MTBC as per manufacturer's instructions. First-line drug susceptibility testing was performed using the proportion method with the MGIT 960 system, according to the manufacturer's instructions. Spoligotyping and region of difference $(\mathrm{RD})$ analysis were performed as previously described (Kamerbeek et al., 1997; Rao et al., 2005). Maf_G67 was grown in $7 \mathrm{H} 9$ liquid media, supplemented with $10 \%$ oleic acid, albumin, dextrose and catalase (OADC) for 7-10 days, with $0.05 \%$ Tween 80 for expansion. When in mid-log phase, bacterial stocks were frozen at $-80^{\circ} \mathrm{C}$ in $1 \mathrm{ml}$ aliquots in the presence of $0.2 \%$ glycerol. Six aliquots were then thawed and used as control for bacterial load determination by CFU enumeration. The number of viable bacteria obtained after 2 weeks of freezing was $1.7 \times 10^{8} \mathrm{CFU} / \mathrm{mL}$, and after 28 weeks of freezing was $1.64 \times 10^{8} \mathrm{CFU} / \mathrm{mL}$. Bacterial stocks were washed twice with PBS before aerosol infections. 


\section{In vitro Cultures and Infections}

Mouse bone marrow-derived macrophages were differentiated from bone marrow precursors in the presence of 20\% L929 cellconditioned media (LCCM). Briefly, bone marrow cells were plated at a concentration of $0.5 \times 10^{6}$ cells $/ \mathrm{mL}$ in Sterilin Petri dishes in $8 \mathrm{~mL}$ of DMEM supplemented with $10 \%$ FCS, Glutamine, HEPES and sodium pyruvate and 20\% LCCM. On day 4, $10 \mathrm{~mL}$ of DMEM with all supplements were added and cells were infected on day 7, at a MOI of 2 (Moreira-Teixeira et al., 2016). THP-1 cells were grown following ATCC instructions, differentiated with $100 \mathrm{nM}$ PMA for $24 \mathrm{~h}$ and infected after a $48 \mathrm{~h}$ rest using a MOI of 1 . Human PBMCs were separated using Histopaque 1077 (Sigma-Aldrich, St. Louis, MO, United States) following the protocol for mononuclear cell separation of SepMate-50 tubes (StemCells, Vancouver, BC, Canada). Cells were frozen and used on the infection day. A MOI of 1 was used, which was calculated to match the total number of cells in culture and which did not compromise cell viability during the infection procedure. At the indicated time points, culture supernatants were harvested and filter-sterilized for cytokine determination by immunoassay (ELISA or Multiplex; eBioscience, Vienna, Austria) or intracellular bacterial load enumeration, as performed previously (Moreira-Teixeira et al., 2016).

\section{Aerosol Infection and Bacterial Burden Determination}

Mice were infected with $M$. africanum via aerosol route using an inhalation exposure system (Glas-Col, United States), as described previously (Moreira-Teixeira et al., 2016). The infection dose was obtained by determining the number of viable bacteria in the lungs of 3-5 mice per experiment, 3 days after infection $(<200 \mathrm{CFU}$ for low doses; $>500 \mathrm{CFU}$ for high doses of infection). For lung bacterial load determination, organs were aseptically excised, individually homogenized, and serial dilutions plated for CFU enumeration (Moreira-Teixeira et al., 2016). CFUs were counted after $21-28$ days incubation at $37^{\circ} \mathrm{C}$.

\section{Histology}

Whole lungs were perfused in situ with PBS. The right upper lobe was excised, fixed in 3.7\% phosphate-buffered formalin for 1 week, embedded in paraffin and cut into 3-mm-thick sections, which were stained with $\mathrm{H} \& \mathrm{E}$, as previously described (Bhatt et al., 2018). Images were acquired with a NanoZoomer 2.0-HT Whole Slide Imager, Digital Pathology Slide Scanner (Hamamatsu, Japan).

\section{Cell Population Analysis by Flow Cytometry}

Lung single cell suspensions were obtained and stained for surface antigens for $30 \mathrm{~min}$ at $4^{\circ} \mathrm{C}$. Stained cells were washed and then fixed overnight in PBS containing 2\% paraformaldehyde. The following antibodies were used: $\alpha \mathrm{CD} 3-\mathrm{PE}$ (clone 145-2C11, eBioscience), $\alpha \mathrm{CD} 4-\mathrm{PB}$ (clone RM4-5, eBioscience), $\alpha \mathrm{CD} 8$-FITC (clone 5H10-1, Biolegend, San Diego, United States), aCD19APC (clone eBio1D3, eBioscience), aLy6G-APC (clone 1A8, Biolegend), $\alpha$ Ly6C-PerCPCy5.5 (clone AL-21, BD Pharmingen), $\alpha \mathrm{CD} 11 \mathrm{~b}-\mathrm{PE}$ (clone M1/70, Biolegend), and $\alpha \mathrm{CD} 11 \mathrm{c}-\mathrm{PB}$ (clone N418, Biolegend). Dead cells were excluded using Zombie Aqua, a viability dye (Biolegend). All samples were analyzed on a CANTO flow cytometer with Diva Software and data analyzed using FlowJo version 10.1.r7 software. The gating strategies used are shown in Supplementary Figure S1.

\section{RNA Extraction and Analysis}

Total RNA from infected lungs was extracted with TRIzol Reagent (Invitrogen, CA, United States), according to the manufacturer's instructions. cDNA was synthesized using the SuperScript First-Strand Synthesis System for RT-PCR (ThermoScientific, United States). Target gene mRNA expression was quantified by real-time PCR using SYBR Green (Thermo Fisher) and normalized to ubiquitin mRNA levels, as described before (Bhatt et al., 2018).

\section{Statistics}

Data were analyzed using GraphPad Prism software, version 8.1.0. Student's $t$ test was used to determine differences between two different groups and one-way ANOVA for more than two groups. Post-tests were applied to multiple comparisons as referred in figure legends. Data was checked for normality and log normality. Differences were considered significant for $p \leq 0.05$ and represented as follows: ${ }^{*} p \leq 0.05{ }^{* *} p \leq 0.01{ }^{* * *} p \leq 0.001$, and ${ }^{* * * *} p \leq 0.0001$.

\section{RESULTS}

\section{Mycobacterium africanum Establishes a Slow Progressing Infection With Minimal Tissue Pathology in Immunocompetent Mice}

The mechanisms underlying the ecological restriction of $M$. africanum remain unknown, but could be explained by a relative lower virulence of $M$. africanum as compared to M. tuberculosis. To gain experimental insight into this question, we decided to investigate the progression of $M$. africanum infection in mice. For this, we used a clinical isolate of M. africanum belonging to L6 (AFRI_I), SIT181, and isolated from a TB patient in Guinea Bissau (Rabna et al., 2015), a country that shows the highest prevalence of $M$. africanum recorded in the African continent (Groenheit et al., 2011). This clinical isolate was susceptible to all five first-line anti-TB drugs. According to the RD typing, Maf_G67 groups in M. africanum subtype II and belongs to the Guinea-Bissau family (absence of RD7, RD8, RD9, and RD10). As expected, the growth of Maf_G67 in axenic medium was slower as compared to M. tuberculosis $\mathrm{H} 37 \mathrm{Rv}$ (Supplementary Figure S2).

We started by infecting macrophages with the M. africanum clinical isolate under study. In vitro infection of mouse BMDM showed persistence of this isolate over time (Figure 1A) and triggered the secretion of both pro- and anti-inflammatory cytokines (Figure 1B). Cytokine secretion was also observed upon infection of PMA-differentiated THP1 cells, a human 

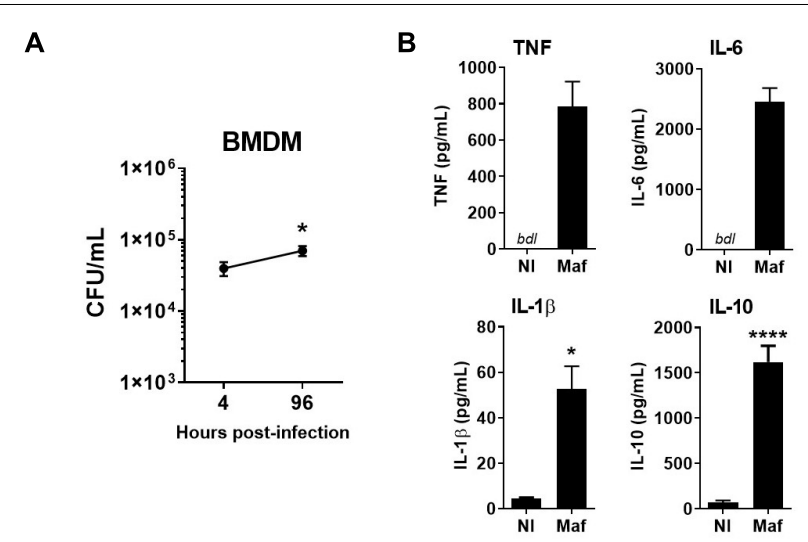

C
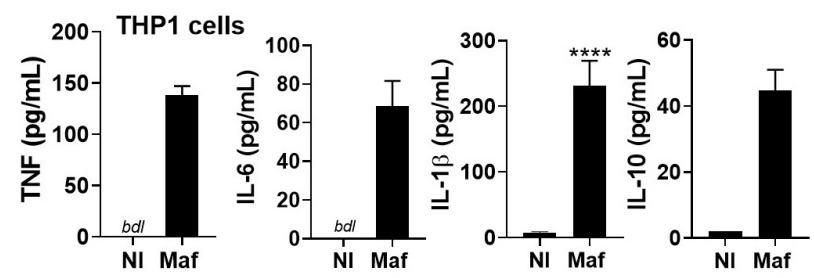

D

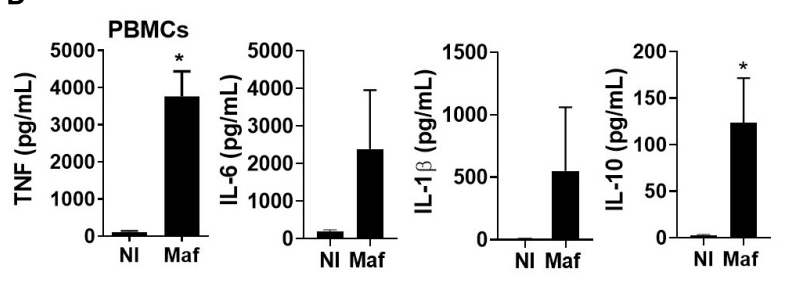

FIGURE 1 | Mycobacterium africanum triggers an immune response in mouse and human cells. $(\mathbf{A}, \mathbf{B}) \mathrm{BMDM}$ were infected with $M$. africanum (Maf) at a $\mathrm{MOI}$ of 2. (A) Four and $96 \mathrm{~h}$ later the intracellular bacterial load was determined by $\mathrm{CFU}$ enumeration. Data is shown as mean $\pm \mathrm{SEM}$ for three independent experiments, each performed in 4-5 replicate wells. (B) Twenty-four hours post-infection, the culture supernatants were harvested and cytokines measured by immunoassay. Data is shown as mean \pm SEM for three independent experiments, each performed in triplicate wells. (C) THP1 cells were differentiated in the presence of PMA, infected with $M$. africanum at a $\mathrm{MOI}$ of 1 and $24 \mathrm{~h}$ later cytokine concentration determined by immunoassay. (D) Human PBMCs were isolated, infected with $M$. africanum at a MOI of 1 and $24 \mathrm{~h}$ later cytokine concentration determined by immunoassay. Data is shown as mean \pm SEM for six independent donours. Non-infected (NI) cells are included as controls. Unpaired $t$-test and Mann-Whitney test were used to perform the statistical analysis. ${ }^{*} p<0.05 ;{ }^{* * *} p<0.0001$. bdl, below detection level.

monocytic cell line (Figure 1C), or human PBMCs (Figure 1D). The rational underlying the use of different cell types was to test if the clinical isolate used in our study triggered a cytokine response that was not dependent on the cell type. These data show that the M. africanum clinical isolate infects, persists in and is recognized by innate immune cells in spite of their different origin and genetic background.

Next, we infected C57BL/6 WT mice with a high dose of M. africanum via the aerosol route. A high dose of infection was performed to probe the host ability to deal with $M$. africanum under more extreme conditions than those associated with low doses of infection. $M$. africanum was able to effectively infect and persist in the mouse lungs, but the bacterial burden did not increase significantly over time (Figure 2A). Moreover, the bacterial burdens detected in the lung were substantially lower than those observed in infections with $M$. tuberculosis reference strains (L4, H37Rv; or L2, HN878) (Table 1). In spite of this poor growth in the lung, $M$. africanum disseminated to the spleen and liver over the 60-day time course (Figure 2A).

Tissue sections of $M$. africanum-infected lungs were examined to assess pulmonary pathology. The extent of inflammatory infiltrates and pathology observed in $M$. africanum-infected animals was minimal (Figure 2B). In the case of $M$. tuberculosis infections, multiple inflammatory foci with infiltration of immune cells and perivascular lymphocyte recruitment was seen (Supplementary Figure S3). These histopathological features, which are characteristic of aerosol infections with high doses of $M$. tuberculosis and accompanied by extensive tissue damage, were mostly inexistent in the case of $M$. africanum infection. On day 30 and 60 post-M. africanum infection, the lung tissue was mostly preserved, except in limited areas where a modest immune infiltration was noted (Figure 2B).

\section{A Discrete Immune Response Develops in the Lung Upon M. africanum Infection}

In parallel, we investigated the recruitment of myeloid and lymphoid immune cell populations to the lung of infected mice. Overall the recruitment of immune cells was observed over time (Figure 2C). Specifically, a significant, but modest, increase was observed in the lungs of infected mice for recruited macrophages, myeloid DCs, neutrophils, CD4, and CD8 T cells (Figure 2C). This observation supports the activation of an immune response upon $M$. africanum infection.

We also measured the transcription of several immune mediators in the lung of $M$. africanum-infected mice. In line with the modest immune cell recruitment observed (Figure 2C), the transcription of the analyzed molecules was also discrete (Figure 2D). Indeed, as compared to non-infected mice, there was no significant upregulation of ifng or $\operatorname{tnf}$ (Figure 2D) and the transcription of nos 2 was undetected (data not shown). A significant upregulation of il17 transcription was observed (Figure 2D). Interestingly, in contrast to what was observed for these pro-inflammatory mediators, the transcription of the antiinflammatory cytokine IL-10 was markedly upregulated during infection (Figure 2D).

Altogether, these data are in line with the bacterial burden and histology findings, which highlight infection in the lung with limited immune activation and pathology.

\section{Lack of IFN- $\gamma$ Does Not Compromise the Survival of Mice Infected With \\ M. africanum}

Given our observations in $M$. africanum-infected C57BL/6 immunocompetent mice, we next questioned whether the absence of a major protective molecule would impact the course of infection by this pathogen. For that, we aerosol-infected 


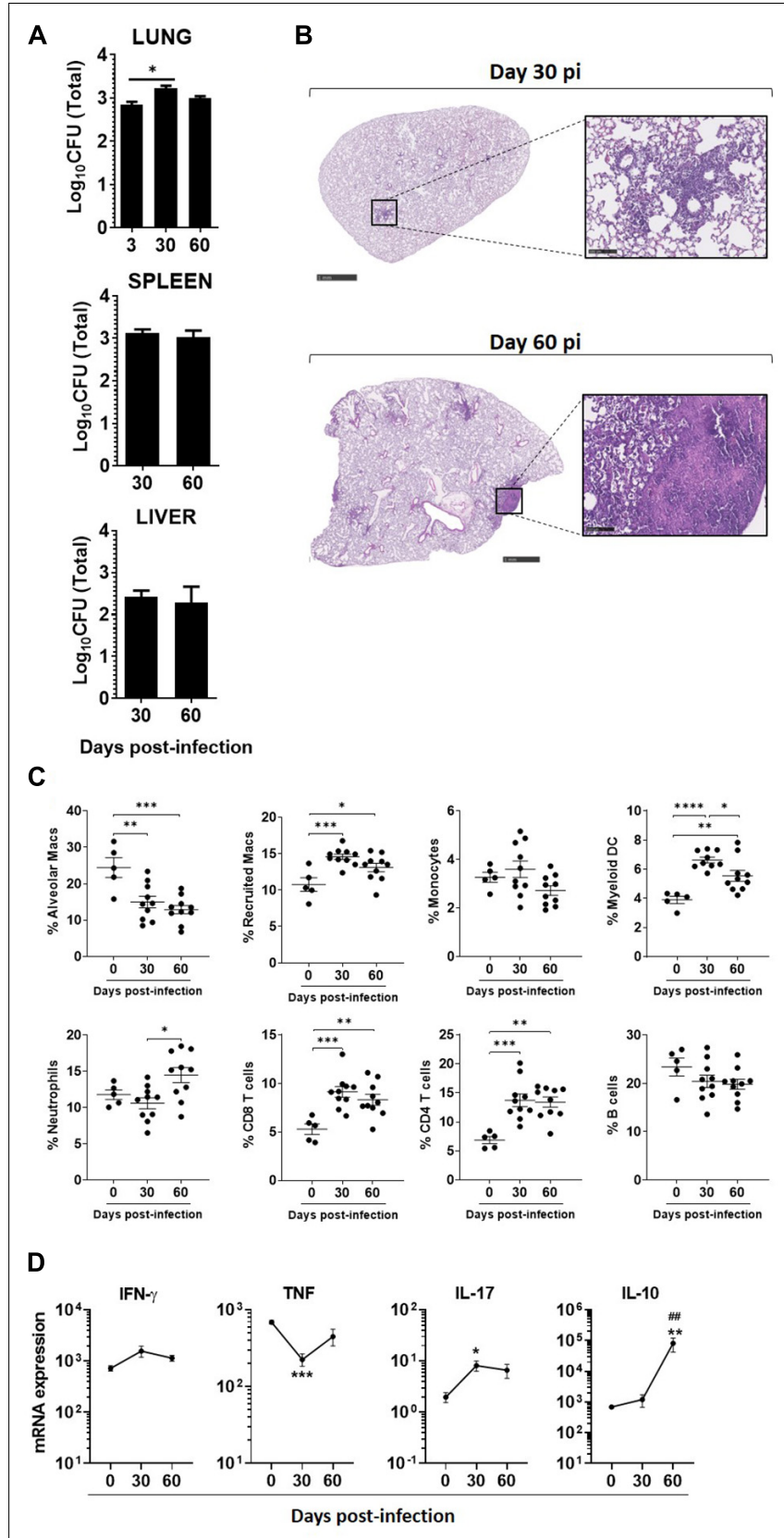

FIGURE 2 | Mycobacterium africanum infection is controlled by immunocompetent hosts, with minimal pathology and immune responses. C57BL/6 mice were infected via aerosol with a high dose (>500 CFU) of M. africanum. (A) At the indicated time points, the lungs, spleens, and livers of infected mice were collected and the bacterial burden determined by CFU enumeration. Lungs were harvested at the indicated time points and (B) pathology determined by H\&E staining; (C) immune cell populations determined by flow cytometry; and (D) the expression of the indicated cytokines measured by real-time PCR. Data are shown as mean \pm SEM from at least two independent experiments with five animals each. The images in (B) are of one animal representative of the experimental group. Scale bar on the left and right images correspond to $1 \mathrm{~mm}$ and $100 \mu \mathrm{m}$, respectively. One-way ANOVA with Tukey's post-test was used to perform the statistical

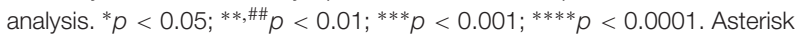
$\left(^{*}\right)$ related to differences compared to $\mathrm{NI}$ mice, and hash (\#) related to differences comparing $d 30$ to $d 60$ post-infection.
TABLE 1 | Comparison of lung bacterial burdens obtained for infections with a M. africanum clinical isolate versus M. tuberculosis reference strains HN878 and H37Rv.

\begin{tabular}{|c|c|c|c|c|}
\hline & \multicolumn{2}{|c|}{ Low dose } & \multicolumn{2}{|c|}{ High dose } \\
\hline & \multicolumn{2}{|c|}{$\log _{10} \mathrm{CFU}$} & \multicolumn{2}{|c|}{$\log _{10} \mathrm{CFU}$} \\
\hline & d30 & d60 & d30 & $d 60$ \\
\hline $\begin{array}{l}\text { M. africanum } \\
\text { (L6) }\end{array}$ & $2.62 \pm 0.022$ & $2.45 \pm 0.021$ & $3.012 \pm 0.129$ & $2.89 \pm 0.068$ \\
\hline $\begin{array}{l}\text { Mtb HN878 } \\
\text { (L2) }\end{array}$ & $6.078 \pm 0.087$ & $5.723 \pm 0.110$ & $7.453 \pm 0.138$ & - \\
\hline $\begin{array}{l}\text { Mtb H37Rv } \\
\text { (L4) }\end{array}$ & $4.866 \pm 0.0898$ & $5.024 \pm 0.296$ & - & $5.203 \pm 0.314$ \\
\hline
\end{tabular}

C57BL/6 mice were infected via aerosol with a low ( $<200$ CFU) or high dose (>500 CFU) of the indicated bacteria and on day 30 or 60 post-infection the lungs were collected and the bacterial burden determined by CFU enumeration. Data are shown as mean \pm SEM from at least five animals per time point. Mtb, M. tuberculosis; $L$, lineage.

C57BL/6 WT or IFN- $\gamma^{-/-}$mice with the M. africanum clinical isolate under study and followed the progression of infection over a period of 90 days. Considering the high susceptibility of IFN- $\gamma^{-/-}$mice to M. tuberculosis (Cooper et al., 1993; Nandi and Behar, 2011; Moreira-Teixeira et al., 2016), we decided to perform the infection with a low dose of bacteria. Notably, IFN- $\gamma^{-/-}$mice infected with $M$. africanum survived for the duration of the experiment, showing no signs of weight loss (Figure 3A). Furthermore, although the lung bacterial burden was significantly increased in IFN- $\gamma^{-/-}$infected mice, as compared to WT ones, bacterial growth was still well controlled (Figure 3B) and far from the typical values observed upon aerosol infection of these mice with M. tuberculosis (Nandi and Behar, 2011; Moreira-Teixeira et al., 2016). As compared to C57BL/6 WT animals, a higher dissemination of $M$. africanum to the spleen and liver of IFN- $\gamma^{-/-}$mice was observed (Figure 3B). Also, in IFN- $\gamma^{-/-}$mice, a robust growth of $M$. africanum was seen in these organs as compared to that observed in the lung. At the histologic level, WT lungs showed no detectable signs of tissue pathology (Figure 3C), showing that as compared to infections with high doses of $M$. africanum (Figure 2B), upon a low dose of infection a lesser infiltration of immune cells develops. IFN- $\gamma^{-1-}$ mice infected with $M$. africanum presented signs of immunopathology in the lung (Figure 3C), with well-localized infiltrates surrounded by areas of healthy tissue.

Collectively, these data suggest that the lack of IFN impacted the ability of mice to deal with $M$. africanum infection, but in contrast to M. tuberculosis infections (Cooper et al., 1993; Nandi and Behar, 2011; Moreira-Teixeira et al., 2016), it did not lead to overt disease and premature death, at least during the first 90 days of infection.

\section{DISCUSSION}

Despite the close relationship between $M$. africanum and M. tuberculosis, their biology, epidemiology, and potential to cause disease is different, calling for the study of TB disease 
A

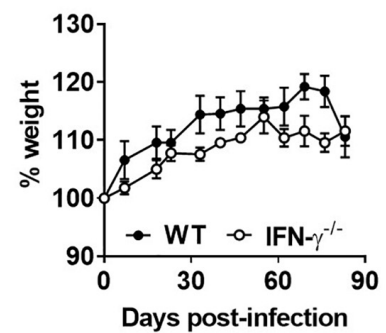

B

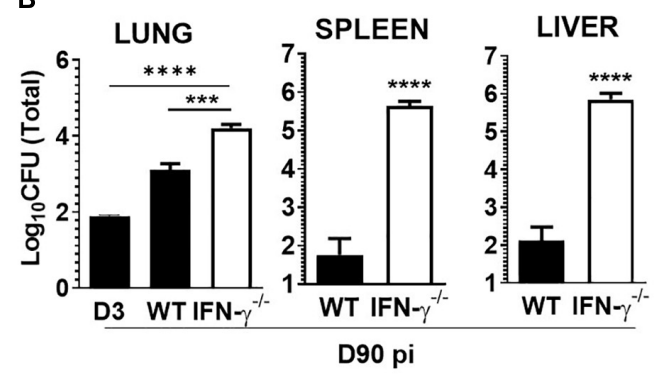

C
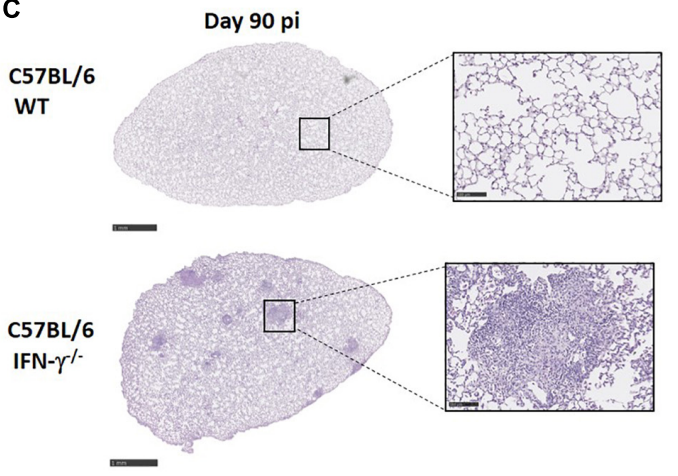

FIGURE 3 | IFN- $\gamma$ is required for optimal control of $M$. africanum, but its absence does not lead to overt disease. C57BL/6 WT (black circles or bars) and IFN- $\gamma^{-1-}$ (white circles or bars) mice were infected by aerosol with a low dose (<200 CFU) of M. africanum. (A) The weight of the animals was monitored weekly up to day 90 . On day 90 post-infection, (B) lungs, spleens, and livers of infected mice were collected and the bacteria burden determined by CFU enumeration and (C) lungs were harvested and pathology determined by H\&E staining. Data are shown as mean \pm SEM from five animals per group. The images in (C) are of one animal representative of the experimental group. Scale bar on the left and right images correspond to $1 \mathrm{~mm}$ and $100 \mu \mathrm{m}$, respectively. Unpaired t-test was used to perform the statistical analysis. ${ }^{* * *} p<0.001 ;{ }^{* * *} p<0.0001$

and associated immune responses to each bacteria. In this study, we resorted to the mouse model to follow the progression of infection by a clinical isolate of M. africanum in terms of bacterial burden, tissue pathology and immune response triggered. Our findings suggest a slow progressing of infection, with mild lung pathology, even in typically highly susceptible hosts. The M. africanum clinical isolate under study infected, persisted and induced a cytokine response by mouse or human cells in in vitro cultures, thus showing its ability to establish infection and to actively interact with host immune cells. The persistence of $M$. africanum in resting macrophages recapitulates previous observations (Homolka et al., 2010).
Our in vivo data are compatible with a slower course of infection of at least some isolates of $M$. africanum in the mouse model, which is in line with what has been reported in humans (de Jong et al., 2008) and also with two previously studies using the mouse model (Bold et al., 2012; Wiens and Ernst, 2016b). In one of these former studies (Bold et al., 2012), the reduction of bacterial burden seen in the lungs of $M$. africanum-infected animals, as compared to M. tuberculosis, was not as pronounced as we report here. In our study and those previously published (Bold et al., 2012; Wiens and Ernst, 2016b), the M. africanum isolates used belonged to L6. However, whereas in our study the clinical isolate has been obtained from a TB patient in Bissau, the previous studies used a bacteria that had been isolated from a TB patient in The Gambia (Bold et al., 2012; Wiens and Ernst, 2016b). It is possible that the variations observed in terms of reported bacterial burdens reflect the recently demonstrated M. africanum intrapathogen diversity (Ates et al., 2018; Otchere et al., 2018), and so that a spectrum of virulence exists among $M$. africanum isolates. It will be important to address this possible spectrum of $M$. africanum virulence, as it may explain several apparent contradictory observations. For example, a possible attenuation of $M$. africanum supports the likely outcompetition of this pathogen by $M$. tuberculosis, as suggested by studies reporting a decreased incidence of $M$. africanum over the time (Koro Koro et al., 2013). However, in other geographic locations, the incidence of $M$. africanum appears to be constant (YeboahManu et al., 2017; Asare et al., 2018) and a recent study including 3580 isolates from 12 different countries (Gehre et al., 2016) show that $M$. africanum, particularly L6, remains a significant cause of TB in West Africa.

Our findings reveal for the first time that infection with a $M$. africanum clinical isolate causes a mild lung pathology upon infection with a high dose of bacteria and over a 60 days period. This is in sharp contrast to what we and others have observed for infections with $M$. tuberculosis isolates and may reflect the slow progression to TB disease described in humans infected with M. africanum (de Jong et al., 2008), and also in some animals (de Jong et al., 2010b). In turn, the slow progression of $M$. africanum infection may be related to the delayed growth described for these bacteria using in vitro methods (Gehre et al., 2013) or to a certain impairment to grow in the lung, reflective of a microaerobic preference developed by $M$. africanum when compared to M. tuberculosis (Ofori-Anyinam et al., 2017). Our observation is important as it suggests that, as compared with M. tuberculosis infection, in humans infected with $M$. africanum, lung damage and eventually cavitation may take a longer time to develop. However, it is important to refer that at presentation the disease caused by $M$. africanum is at least as severe as that caused by M. tuberculosis (de Jong et al., 2007; Tientcheu et al., 2016). Still, a slower progression to disease may eventually compromise the transmission of $M$. africanum in the long term. Indeed, a recent study showed that in Ghana the transmission of $M$. africanum seems to be lower than that of $M$. tuberculosis (Asare et al., 2018), whereas in Mali person to person recent transmission has been reported (Winglee et al., 2016) and in The Gambia similar rates of transmission between $M$. tuberculosis and M. africanum have been described (de Jong et al., 2008). 
These variations may be related to the host and pathogen intrinsic diversity.

Furthermore, we show the dissemination of this M. africanum isolate to the spleen and liver of infected animals, where it appears to grow somehow better than in the lung. Several reasons may explain the dissemination of a bacteria that is not growing well in the lung. On one hand, it is possible that the lack of a robust $\mathrm{T}$ cell response may allow the escape of more bacteria from the lung to other organs. In HIV-TB coinfected patients, where CD4 $\mathrm{T}$ cell responses are lacking, a higher dissemination of M. tuberculosis occurs (Aaron et al., 2004). On the other hand, the more competent growth of $M$. africanum in the spleen and liver, as compared to the lung, may be a consequence of the downregulation of DosR and preference for microaerobic growth described for this bacteria (Ofori-Anyinam et al., 2017). In conclusion, our observations offer the hypothesis that TB caused by $M$. africanum may be associated with higher rates of extra-pulmonary or disseminated disease, as suggested in a recent study (Sharma et al., 2016). They also indicate a reduced degree of virulence of $M$. africanum, which may be related to dissemination, rather than lung damage, during early stages of infection.

Moreover, the control of this $M$. africanum isolate in the mouse lung is reflected in the low recruitment of immune cells and induction of cytokine transcription. Indeed, the transcription of key cytokines as IFN- $\gamma$, IL-17, and TNF, as well as NOS2, in the lungs of infected over non-infected mice was barely detected. Noteworthy, however, was the robust transcription of IL-10, which production was also seen in in vitro infected mouse or human macrophages, and human PBMCs. It will be interesting to, in the future, further dissect the mechanisms regulating IL-10 expression upon infections with different isolates of $M$. africanum, as well as to elucidate the possible contribution of IL-10 for $M$. africanum pathogenesis, both in the context of immunocompetent or immunocompromised mice. Furthermore, it will be also interesting to establish the dynamic of immune cell recruitment during the initial phases of infection, when the innate immune responses are likely predominant. Another outstanding question relates to the mechanisms underlying the lower virulence associated with $M$. africanum, which remain undisclosed. Of note, the interaction of $M$. africanum with mouse macrophages has been shown to differ from that of $M$. tuberculosis, in what regards the transcriptional reprograming of the bacteria (Homolka et al., 2010), as well as the triggering of mitochondrial ROS and ultimately of type I IFN (Wiens and Ernst, 2016a). How these observations articulate and whether they may contribute to the lower virulence of $M$. africanum is unknown.

The overwhelming protective nature of IFN- $\gamma$ in TB has been extensively described in mice and humans. IFN $-\gamma^{-/-}$mice infected with $M$. tuberculosis typically succumb to infection during the first 40 days (Cooper et al., 1993; Nandi and Behar, 2011; Moreira-Teixeira et al., 2016), whereas humans with mutations in the IFN- $\gamma$ producing/responding axis present Mendelian susceptibility to mycobacterial disease (Abel et al., 2018). Thus, the finding presented here for the first time that mice lacking IFN- $\gamma$ coped well with $M$. africanum aerosol infection was most surprising. Still, it is important to refer that although IFN $-\gamma^{-/}$mice survived a low dose infection with this isolate of M. africanum for at least 90 days, they did present higher lung bacterial burden and evidence for immune pathology, not seen in IFN- $\gamma$ competent mice. This indicates that similar protective mechanisms operate among humanadapted TB causing bacteria, albeit their required threshold and dynamics for host protection are different. In the same context, a recent report demonstrated that type I IFN receptor signaling is detrimental during infections with a different isolate of M. africanum (Wiens and Ernst, 2016b), as previously reported for M. tuberculosis (Berry et al., 2010; Mayer-Barber et al., 2014). Also, for both TB patients and household contacts, $M$. africanum infection has been shown to elicit diminished $\mathrm{T}$ cell responses to ESAT6, as compared to those elicited by M. tuberculosis (de Jong et al., 2006). A subsequent study identified higher proportion of single TNF and lower proportion of single-IL-2-producing CD4 and CD8 T cells in M. africanuminfected patients, as compared with $M$. tuberculosis-infected ones before antibiotherapy, and persistently high proportion of activated T cells in $M$. africanum infected patients after treatment (Tientcheu et al., 2014).

In conclusion, several recent studies, including the one presented here, are bridging the gap relatively to our limited knowledge on $M$. africanum and pointing to an overall lower virulence of these bacteria. The mechanisms underlying this lower virulence are not fully understood, highlighting the need for more research using diverse M. africanum isolates, including those belonging to L5. Understanding whether $M$. africanum is indeed less virulent and the underlying causes, may shed important clues on how to «attenuate» M. tuberculosis, either by targeting the bacteria or the host immune response (Zumla et al., 2017). Furthermore, a deep understanding of $M$. africanum infections will inform the design of common or specific vaccines and therapies.

\section{DATA AVAILABILITY}

The datasets generated for this study are available on request to the corresponding author.

\section{ETHICS STATEMENT}

The human study that originated the blood samples for peripheral blood mononuclear cells (PBMC) isolation was reviewed and approved by the Portuguese Comissão de Ética para a Saúde da ARS Norte (project T792). The patients/participants provided their written informed consent to participate in this study. Animal experiments were performed in strict accordance with the recommendations of the EU Directive 2010/63/EU and approved by the Portuguese National Authority for Animal Health (DGAV-Ref. 0421/000/000/2016). 


\section{AUTHOR CONTRIBUTIONS}

BC, KF, JS, AM, and DM performed the experiments. BC and KF analyzed the data and wrote the manuscript. LS, PRa, and MV performed the work leading to the isolation of the clinical strain of M. africanum used. PRo and MV wrote the manuscript. MS supervised the study, planned the experiments, analyzed the data, and wrote the manuscript.

\section{FUNDING}

This work was supported by Fundo Europeu de Desenvolvimento Regional funds through the COMPETE 2020 - Operational Programme for Competitiveness and Internationalization (POCI), Portugal 2020, and by Portuguese funds through Fundação para a Ciência e a Tecnologia, Ministério da Ciência, Tecnologia e Inovação in the framework of the project "Institute for Research and Innovation in Health Sciences" (POCI-01-0145-FEDER-007274), and by grants FCT - Aga Khan Development Network (ref 333197025), POCI-01-0145FEDER-028955 (to MS), PTDC/BIA-MIC/30692/2017, and UID/Multi/04413/2013 (to DM and MV). BC and KF were funded by FCT Ph.D. scholarships SFRH/BD/114403/2016 and SFRH/BD/114405/2016, respectively. The Gulbenkian Foundation is acknowledged for a field work research grant to $\mathrm{BC}$, Bolsas de apoio à investigação para estudantes de doutoramento dos PALOP, Ref. P-146397. DM and MS

\section{REFERENCES}

Aaron, L., Saadoun, D., Calatroni, I., Launay, O., Memain, N., Vincent, V., et al. (2004). Tuberculosis in HIV-infected patients: a comprehensive review. Clin. Microbiol. Infect. 10, 388-398. doi: 10.1111/j.1469-0691.2004. 00758.x

Abel, L., Fellay, J., Haas, D. W., Schurr, E., Srikrishna, G., Urbanowski, M., et al. (2018). Genetics of human susceptibility to active and latent tuberculosis: present knowledge and future perspectives. Lancet Infect. Dis. 18, e64-e75. doi: 10.1016/S1473-3099(17)30623-0

Alfredsen, S., and Saxegaard, F. (1992). An outbreak of tuberculosis in pigs and cattle caused by Mycobacterium africanum. Vet. Rec. 131, 51-53. doi: 10.1136/ vr.131.3.51

Asante-Poku, A., Yeboah-Manu, D., Otchere, I. D., Aboagye, S. Y., Stucki, D., Hattendorf, J., et al. (2015). Mycobacterium africanum is associated with patient ethnicity in Ghana. PLoS Negl. Trop. Dis. 9:e3370. doi: 10.1371/journal.pntd. 0003370

Asare, P., Asante-Poku, A., Prah, D. A., Borrell, S., Osei-Wusu, S., Otchere, I. D., et al. (2018). Reduced transmission of Mycobacterium africanum compared to Mycobacterium tuberculosis in urban West Africa. Int. J. Infect. Dis. 73, 30-42. doi: 10.1016/j.ijid.2018.05.014

Ates, L. S., Dippenaar, A., Sayes, F., Pawlik, A., Bouchier, C., Ma, L., et al. (2018). Unexpected genomic and phenotypic diversity of Mycobacterium africanum lineage 5 affects drug resistance, protein secretion, and immunogenicity. Genome Biol. Evol. 10, 1858-1874. doi: 10.1093/gbe/evy145

Bastos, H. N., Osorio, N. S., Gagneux, S., Comas, I., and Saraiva, M. (2017). The troika host-pathogen-extrinsic factors in tuberculosis: modulating inflammation and clinical outcomes. Front. Immunol. 8:1948. doi: 10.3389/ fimmu.2017.01948

Berry, M. P., Graham, C. M., McNab, F. W., Xu, Z., Bloch, S. A., Oni, T., et al. (2010). An interferon-inducible neutrophil-driven blood transcriptional signature in human tuberculosis. Nature 466, 973-977. doi: 10.1038/nature0 9247 were supported by FCT through Estimulo Individual ao Emprego Científico.

\section{ACKNOWLEDGMENTS}

The authors thank the excellent support from the i3S scientific platforms, namely Animal Facility and Translational Cytometry.

\section{SUPPLEMENTARY MATERIAL}

The Supplementary Material for this article can be found online at: https://www.frontiersin.org/articles/10.3389/fmicb. 2019.02102/full\#supplementary-material

FIGURE S1 | Gating strategy used to delineate the cellular populations present in the lung.

FIGURE S2 | In vitro growth rates of $M$. africanum G67 compared to M. tuberculosis H37Rv. Bacterial suspensions were adjusted to $10^{6} \mathrm{CFU} / \mathrm{ml}$ with a sterile saline solution and $0.5 \mathrm{ml}$ transferred into a MGIT tube containing $10 \%$ OADC. Growth rates were measured at $37^{\circ} \mathrm{C}$ with the MGIT 960 and the Epicenter software for 28 days.

FIGURE S3 | Infection of C57BL/6 mice with a high dose of $M$. tuberculosis. C57BL/6 mice were infected via aerosol with a high dose (>500 CFU) of M. tuberculosis strain HN878. On day 30 post-infection, the lungs were collected and pathology determined by H\&E staining. The images are of one animal representative of the experimental group $(n=5)$. Scale bar corresponds to $2.5 \mathrm{~mm}$ on the left and $100 \mu \mathrm{m}$ on the right panel.

Bhatt, K., Machado, H., Osorio, N. S., Sousa, J., Cardoso, F., Magalhaes, C., et al. (2018). A Nonribosomal peptide synthase gene driving virulence in Mycobacterium tuberculosis. mSphere 3:e00352-18. doi: 10.1128/mSphere. 00352- 18

Bold, T. D., Davis, D. C., Penberthy, K. K., Cox, L. M., Ernst, J. D., and de Jong, B. C. (2012). Impaired fitness of Mycobacterium africanum despite secretion of ESAT-6. J. Infect. Dis. 205, 984-990. doi: 10.1093/infdis/jir883

Brites, D., and Gagneux, S. (2015). Co-evolution of Mycobacterium tuberculosis and homo sapiens. Immunol. Rev. 264, 6-24. doi: 10.1111/imr.12264

Cooper, A. M., Dalton, D. K., Stewart, T. A., Griffin, J. P., Russell, D. G., and Orme, I. M. (1993). Disseminated tuberculosis in interferon gamma gene-disrupted mice. J. Exp. Med. 178, 2243-2247. doi: 10.1084/jem.178.6.2243

Coscolla, M., Lewin, A., Metzger, S., Maetz-Rennsing, K., Calvignac-Spencer, S., Nitsche, A., et al. (2013). Novel Mycobacterium tuberculosis complex isolate from a wild chimpanzee. Emerg. Infect. Dis. 19, 969-976. doi: 10.3201/eid1906. 121012

de Jong, B. C., Adetifa, I., Walther, B., Hill, P. C., Antonio, M., Ota, M., et al. (2010a). Differences between tuberculosis cases infected with Mycobacterium africanum, West African type 2, relative to Euro-American Mycobacterium tuberculosis: an update. FEMS Immunol. Med. Microbiol. 58, 102-105. doi: 10.1111/j.1574-695X.2009.00628.x

de Jong, B. C., Antonio, M., and Gagneux, S. (2010b). Mycobacterium africanumreview of an important cause of human tuberculosis in West Africa. PLoS Negl. Trop. Dis. 28 4:e744. doi: 10.1371/journal.pntd.0000744

de Jong, B. C., Hill, P. C., Aiken, A., Awine, T., Antonio, M., Adetifa, I. M., et al. (2008). Progression to active tuberculosis, but not transmission, varies by Mycobacterium tuberculosis lineage in the Gambia. J. Infect. Dis. 198, 1037-1043. doi: 10.1086/591504

de Jong, B. C., Hill, P. C., Aiken, A., Jeffries, D. J., Onipede, A., Small, P. M., et al. (2007). Clinical presentation and outcome of tuberculosis patients infected by M. africanum versus M. tuberculosis. Int. J. Tuberc. Lung Dis. 11, 450-456.

de Jong, B. C., Hill, P. C., Brookes, R. H., Gagneux, S., Jeffries, D. J., Otu, J. K., et al. (2006). Mycobacterium africanum elicits an attenuated T cell response to 
early secreted antigenic target, $6 \mathrm{kDa}$, in patients with tuberculosis and their household contacts. J. Infect. Dis. 193, 1279-1286. doi: 10.1086/502977

Dosso, M., Bonard, D., Msellati, P., Bamba, A., Doulhourou, C., Vincent, V., et al. (1999). Primary resistance to antituberculosis drugs: a national survey conducted in Cote d'Ivoire in 1995-1996. ivoirian study group on tuberculosis resistance. Int. J. Tuberc. Lung Dis. 3, 805-809.

Gagneux, S. (2018). Ecology and evolution of Mycobacterium tuberculosis. Nat. Rev. Microbiol. 16, 202-213. doi: 10.1038/nrmicro.2018.8

Gehre, F., Kumar, S., Kendall, L., Ejo, M., Secka, O., Ofori-Anyinam, B., et al. (2016). A Mycobacterial perspective on Tuberculosis in West Africa: significant geographical variation of $M$. africanum and Other $M$. tuberculosis complex lineages. PLoS Negl. Trop. Dis. 10:e0004408. doi: 10.1371/journal.pntd.0004408

Gehre, F., Otu, J., DeRiemer, K., de Sessions, P. F., Hibberd, M. L., Mulders, W., et al. (2013). Deciphering the growth behaviour of Mycobacterium africanum. PLoS Negl. Trop. Dis. 7:e2220. doi: 10.1371/journal.pntd.0002220

Gonzalo-Asensio, J., Malaga, W., Pawlik, A., Astarie-Dequeker, C., Passemar, C., Moreau, F., et al. (2014). Evolutionary history of tuberculosis shaped by conserved mutations in the PhoPR virulence regulator. Proc. Natl. Acad. Sci. U.S.A. 111, 11491-11496. doi: 10.1073/pnas.1406693111

Groenheit, R., Ghebremichael, S., Svensson, J., Rabna, P., Colombatti, R., Riccardi, F., et al. (2011). The Guinea-Bissau family of Mycobacterium tuberculosis complex revisited. PLoS One 6:e18601. doi: 10.1371/journal.pone.0018601

Gudan, A., Artukovic, B., Cvetnic, Z., Spicic, S., Beck, A., Hohsteter, M., et al. (2008). Disseminated tuberculosis in hyrax (Procavia capensis) caused by Mycobacterium africanum. J. Zoo Wildl. Med. 39, 386-391. doi: 10.1638/06041.1

Homolka, S., Niemann, S., Russell, D. G., and Rohde, K. H. (2010). Functional genetic diversity among Mycobacterium tuberculosis complex clinical isolates: delineation of conserved core and lineage-specific transcriptomes during intracellular survival. PLoS Pathog. 6:e1000988. doi: 10.1371/journal.ppat. 1000988

Isea-Pena, M. C., Brezmes-Valdivieso, M. F., Gonzalez-Velasco, M. C., LezcanoCarrera, M. A., Lopez-Urrutia-Lorente, L., Martin-Casabona, N., et al. (2012). Mycobacterium africanum, an emerging disease in high-income countries? Int. J. Tuberc. Lung Dis. 16, 1400-1404. doi: 10.5588/ijtld.12.0142

Kallenius, G., Koivula, T., Ghebremichael, S., Hoffner, S. E., Norberg, R., Svensson, E., et al. (1999). Evolution and clonal traits of Mycobacterium tuberculosis complex in Guinea-Bissau. J. Clin. Microbiol. 37, 3872-3878.

Kamerbeek, J., Schouls, L., Kolk, A., van Agterveld, M., van Soolingen, D., Kuijper, S., et al. (1997). Simultaneous detection and strain differentiation of Mycobacterium tuberculosis for diagnosis and epidemiology. J. Clin. Microbiol. 35, 907-914.

Koro Koro, F., Kamdem Simo, Y., Piam, F. F., Noeske, J., Gutierrez, C., Kuaban, C., et al. (2013). Population dynamics of tuberculous Bacilli in Cameroon as assessed by spoligotyping. J. Clin. Microbiol. 51, 299-302. doi: 10.1128/JCM. 01196- 12

Mayer-Barber, K. D., Andrade, B. B., Oland, S. D., Amaral, E. P., Barber, D. L., Gonzales, J., et al. (2014). Host-directed therapy of tuberculosis based on interleukin-1 and type I interferon crosstalk. Nature 511, 99-103. doi: 10.1038/ nature13489

Meyer, C. G., Scarisbrick, G., Niemann, S., Browne, E. N., Chinbuah, M. A., Gyapong, J., et al. (2008). Pulmonary tuberculosis: virulence of Mycobacterium africanum and relevance in HIV co-infection. Tuberculosis 88, 482-489. doi: 10.1016/j.tube.2008.05.004

Moreira-Teixeira, L., Sousa, J., McNab, F. W., Torrado, E., Cardoso, F., Machado, H., et al. (2016). Type I IFN inhibits alternative macrophage activation during Mycobacterium tuberculosis infection and leads to enhanced protection in the absence of IFN-gamma signaling. J. Immunol. 197, 4714-4726. doi: 10.4049/ jimmunol.1600584

Nandi, B., and Behar, S. M. (2011). Regulation of neutrophils by interferongamma limits lung inflammation during tuberculosis infection. J. Exp. Med. 208, 2251-2262. doi: 10.1084/jem.20110919

Ofori-Anyinam, B., Dolganov, G., Van, T., Davis, J. L., Walter, N. D., Garcia, B. J., et al. (2017). Significant under expression of the DosR regulon in M. tuberculosis complex lineage 6 in sputum. Tuberculosis 104, 58-64. doi: 10.1016/j.tube.2017. 03.001

Otchere, I. D., Coscolla, M., Sanchez-Buso, L., Asante-Poku, A., Brites, D., Loiseau, C., et al. (2018). Comparative genomics of Mycobacterium africanum lineage 5 and lineage 6 from ghana suggests distinct ecological niches. Sci. Rep. 8:11269. doi: 10.1038/s41598-018-29620-2

Rabna, P., Ramos, J., Ponce, G., Sanca, L., Mané, M., Armada, A., et al. (2015). Direct detection by the Xpert MTB/RIF assay and characterization of multi and poly drug-resistant tuberculosis in guineabissau. West Africa. PLoS One 10:e0127536. doi: 10.1371/journal.pone.012 7536

Rao, K. R., Kauser, F., Srinivas, S., Zanetti, S., Sechi, L. A., Ahmed, N., et al. (2005). Analysis of genomic downsizing on the basis of region-of-difference polymorphism profiling of Mycobacterium tuberculosis patient isolates reveals geographic partitioning. J. Clin. Microbiol. 43, 5978-5982. doi: 10.1128/JCM.43. 12.5978-5982.2005

Sharma, A., Bloss, E., Heilig, C. M., and Click, E. S. (2016). Tuberculosis caused by Mycobacterium africanum, United States, 2004-2013. Emerg. Infect. Dis. 22, 396-403. doi: 10.3201/eid2203.151505

Thorel, M. F. (1980). Isolation of Mycobacterium africanum from monkeys. Tubercle 61, 101-104. doi: 10.1016/0041-3879(80)90018-5

Tientcheu, L. D., Haks, M. C., Agbla, S. C., Sutherland, J. S., Adetifa, I. M., Donkor, S., et al. (2016). Host Immune responses differ between M. africanumand M. tuberculosis-infected patients following standard anti-tuberculosis treatment. PLoS Negl. Trop. Dis. 10:e0004701. doi: 10.1371/journal.pntd. 0004701

Tientcheu, L. D., Sutherland, J. S., de Jong, B. C., Kampmann, B., Jafali, J., Adetifa, I. M., et al. (2014). Differences in T-cell responses between Mycobacterium tuberculosis and Mycobacterium africanum-infected patients. Eur. J. Immunol. 44, 1387-1398. doi: 10.1002/eji.201343956

WHO, (2018). Global Tuberculosis Control- Report. Geneva: World Health Organization.

Wiens, K. E., and Ernst, J. D. (2016a). The mechanism for type I interferon induction by Mycobacterium tuberculosis is bacterial strain-dependent. PLoS Pathog. 12:e1005809. doi: 10.1371/journal.ppat.1005809

Wiens, K. E., and Ernst, J. D. (2016b). Type I interferon is pathogenic during chronic Mycobacterium africanum infection. J. Infect. Dis. 214, 1893-1896. doi: 10.1093/infdis/jiw519

Winglee, K., Manson McGuire, A., Maiga, M., Abeel, T., Shea, T., Desjardins, C. A., et al. (2016). Whole genome sequencing of Mycobacterium africanum strains from mali provides insights into the mechanisms of geographic restriction. PLoS Negl. Trop. Dis. 10:e0004332. doi: 10.1371/journal.pntd.00 04332

Yeboah-Manu, D., de Jong, B. C., and Gehre, F. (2017). The biology and epidemiology of Mycobacterium africanum. Adv. Exp. Med. Biol. 1019, 117-133. doi: 10.1007/978-3-319-64371-7_6

Zumla, A., Otchere, I. D., Mensah, G. I., Asante-Poku, A., Gehre, F., Maeurer M., et al. (2017). Learning from epidemiological, clinical, and immunological studies on Mycobacterium africanum for improving current understanding of host-pathogen interactions, and for the development and evaluation of diagnostics, host-directed therapies, and vaccines for tuberculosis. Int. J. Infect. Dis. 56, 126-129. doi: 10.1016/j.ijid.2016.12.003

Conflict of Interest Statement: The authors declare that the research was conducted in the absence of any commercial or financial relationships that could be construed as a potential conflict of interest.

Copyright () 2019 Cá, Fonseca, Sousa, Maceiras, Machado, Sanca, Rabna, Rodrigues, Viveiros and Saraiva. This is an open-access article distributed under the terms of the Creative Commons Attribution License (CC BY). The use, distribution or reproduction in other forums is permitted, provided the original author(s) and the copyright owner(s) are credited and that the original publication in this journal is cited, in accordance with accepted academic practice. No use, distribution or reproduction is permitted which does not comply with these terms. 